\title{
The HeartLander: A novel epicardial crawling robot for myocardial injections
}

\author{
N.A. Patronik ${ }^{\mathrm{a}, *}$, C.N. Riviere ${ }^{\mathrm{a}}$, S. El Qarra ${ }^{\mathrm{b}}$, M.A. Zenati ${ }^{\mathrm{a}, \mathrm{b}}$ \\ ${ }^{\mathrm{a}}$ The Robotics Institute, Carnegie Mellon University, USA \\ ${ }^{\mathrm{b}}$ Division of Cardiothoracic Surgery, University of Pittsburgh, USA
}

\begin{abstract}
Myocardial infarction is the leading cause of congestive heart failure and death in the industrialized world. Stem cell transplantation to failing myocardium appears to improve heart function following myocardial infarction, but further refinement of the delivery methodology is required. The HeartLander miniature mobile robot has the ability to adhere to the epicardium, travel to the operative site, and perform intramyocardial injections under direct control of the surgeon. This paradigm obviates sternotomy, cardiopulmonary bypass, mechanical stabilization and lung deflation, while granting improved access. To facilitate movement under the pericardium, a small prototype with a tapered front has been constructed that is $11 \mathrm{~mm}$ tall and fits through a $15-\mathrm{mm}$ cannula. This prototype was tested in beating-heart porcine trials via median sternotomy, but with the pericardium intact $(N=2)$. The HeartLander was able to maintain prehension and travel without being displaced by the overhead motion of the pericardium. Myocardial injections of tissue dye were performed successfully at several locations. These experiments show the feasibility of navigating under the pericardium and performing needle injections into the myocardium using video feedback. Future research will move toward minimally invasive testing without sternotomy, including both locomotion and therapy. (C) 2005 CARS \& Elsevier B.V. All rights reserved.
\end{abstract}

Keywords: Mobile robot; Cardiac surgery; Beating heart; Minimally invasive; Myocardial injection; Stem cell

\section{Introduction}

Congestive heart failure caused by myocardial infarction is the leading cause of death in the industrialized world [1]. The severity of this disease is illustrated by the fact that $50 \%$ of congestive heart failure patients die within 5 years of diagnosis [2]. Because

\footnotetext{
* Corresponding author.

E-mail address: patronik@cmu.edu (N.A. Patronik).
} 
cardiomyocytes are terminally differentiated, there are currently no effective means to replace scarred myocardium with viable functioning myocardium. Stem cells are precursor cells that are capable of proliferation, self-renewal, and differentiation into cardiomyocytes. Cardiomyoplasty, the injection of stem cells into failing myocardium, offers the potential to reverse the deleterious hemodynamic and neurohormonal effects that result from myocardial infarction and lead to congestive heart failure [1]. Although clinical data on the effects of cardiomyoplasty are very limited, the results of preclinical animal studies and early clinical investigations have shown regenerated myocardium and increased perfusion in the infarct area leading to improved cardiac function [3-5].

There are three general delivery methods currently used in cardiomyoplasty, the effectiveness of which have yet to be quantitatively determined. The most invasive technique is intramyocardial delivery, where stem cells are injected directly into the contracting myocardium wall bordering the infarct. This method allows direct visualization of the target zone, and is the preferred approach when the patient is already undergoing an open cardiac procedure [1]. Less invasive techniques include intracoronary and intravascular delivery, which must be further developed to increase the localization of treatment to the targeted myocardium [1]. Recently, the first minimally invasive intramyocardial injections were performed through 4 small incisions made in the chest [5]. As testing of stem cell transplantation matures, enhanced delivery methods that provide superior access in a less invasive manner will become essential.

HeartLander is a miniature mobile robot that is designed to facilitate minimally invasive, beating-heart intrapericardial therapy [6]. The robot can be introduced into the thoracic cavity through a subxiphoid approach, and inserted on the epicardial surface though a small incision in the pericardium made with a subxiphoid videopericardioscopic (SVP) device [7]. HeartLander can then adhere to the epicardium using suction, and travel to any location for intervention under direction control of the surgeon. This novel paradigm obviates median sternotomy, cardiopulmonary bypass, mechanical cardiac stabilization, access limitations, and lung deflation. HeartLander will be capable of providing a stable platform from which to perform intramyocardial injection of stem cells at any location on the heart while reducing the morbidity associated with gaining access.

\section{Material and methods}

The HeartLander crawling robot is connected via tether to supporting tabletop instrumentation that includes motors for actuation, a pump to supply vacuum pressure, and a computer for visual feedback and control. This design allows the therapeutic portion of the robot to be passive, lightweight, inexpensive, and therefore disposable in clinical use. The crawling robot (shown in Fig. 1) consists of two body sections that can independently adhere to the epicardium and translate during locomotion. Each body section is $11 \mathrm{~mm}$ tall and $10 \mathrm{~mm}$ wide, thus allowing it to pass through a $15-\mathrm{mm}$ standard port. The tapered front end aids in maneuvering beneath the pericardium, and provides visualization of the epicardium directly in front of the robot.

This prototype has the ability to perform myocardial injections using a custom $27 \mathrm{G}$ needle that passes through a sheath in the working channel. When the target area is located using video feedback, the surgeon advances the needle by hand, through the sheath, into 


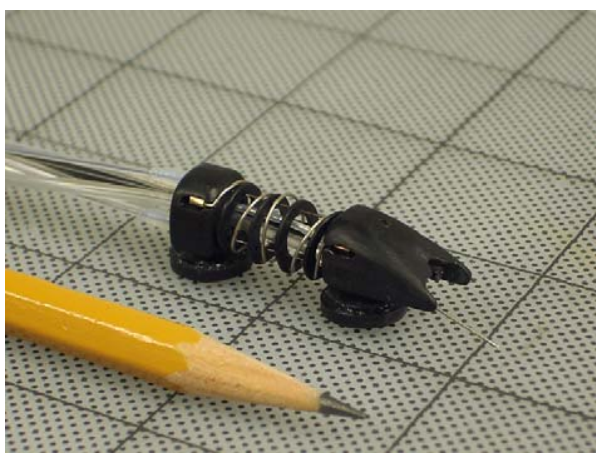

A

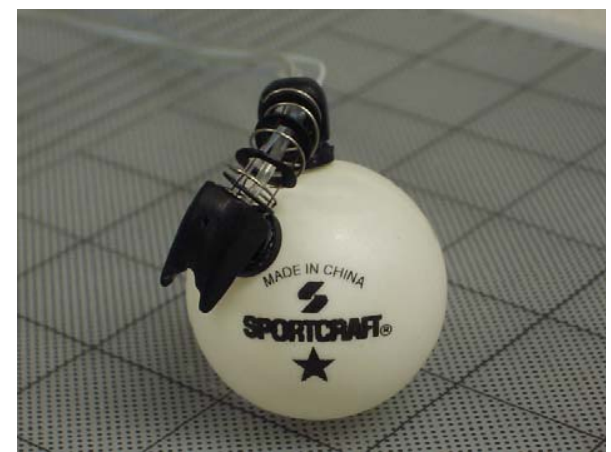

B

Fig. 1. (A) The HeartLander prototype, with a standard pencil shown for scale. The robot is $11 \mathrm{~mm}$ tall and 10 $\mathrm{mm}$ wide. The needle for myocardial injection can be seen protruding from the front body section. (B) The robot adapting to the curved surface of a standard 40-mm diameter table tennis ball. The robot is actuated by three wires running through the tether, which provides two rotational and one translational degrees of freedom.

the tissue. The surgeon relies on both tactile sensation from the needle and visual feedback on the video monitor to judge the depth of the puncture. The injection is then performed using a syringe attached to the proximal end of the needle.

HeartLander adheres to the epicardium using suction, supplied to the suction pads through vacuum lines in the tether. The external pump provides a vacuum pressure no greater than $0.053 \mathrm{~N} / \mathrm{mm}^{2}$ (400 $\mathrm{mm} \mathrm{Hg}$ ), which has been found to be effective and safe for use in FDA-approved cardiac stabilizers [8]. During locomotion, the vacuum pressure is monitored by pressure sensors and regulated by computer-controlled valves, both located in the supporting instrumentation.

Actuation for locomotion is provided by three nitinol wires that pass through the tether and are driven by motor belts in the supporting instrumentation. The locomotion of HeartLander is a cyclic, inchworm-like process that requires coordination of the motors and pressure valves [6]. This process is controlled by the supporting computer with input from a joystick interface, thus making the mechanical details transparent to the user. The seal at each suction pad is monitored by the pressure sensors to ensure that a good grip of the epicardium is maintained throughout the locomotive cycle. If the active suction pad does not achieve a tight seal, the robot automatically adapts to the surface curvature using its two rotational degrees of freedom (see Fig 1B).

Visual feedback from the front body is relayed to an external video camera by a 1.6- $\mathrm{mm}$ diameter flexible fiber optic endoscope running through the tether, and displayed to the user on the monitor.

\section{Testing}

Large (30-45 kg) crossbred swine were used for all trials. After standard single-lumen endotracheal intubation, a surgical plane of anesthesia was maintained using isoflurane, 1$3 \%$. Each animal was placed into supine position and median sternotomy was performed. The pericardium was left intact. Invasive hemodynamic and arterial blood gas monitoring was performed throughout the procedure. 

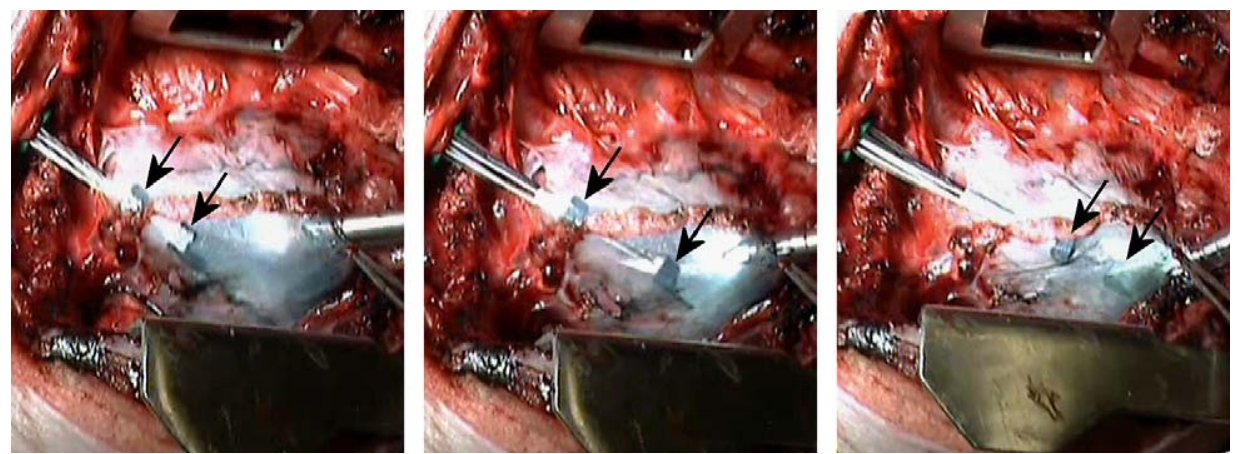

Fig. 2. A time series of photographs showing HeartLander walking across the epicardium beneath the pericardium. The arrows highlight the rear and front body sections. HeartLander was introduced into the thoracic cavity through a $15-\mathrm{mm}$ port, and inserted on the epicardium through a $10-\mathrm{mm}$ slit in the pericardium.

HeartLander was introduced into the thoracic cavity through a 15-mm port (see Fig. 2), simulating a subxiphoid incision, and applied to the epicardial surface through a $10-\mathrm{mm}$ slit in the pericardium. The device was able to maintain prehension of the epicardium despite constant overhead contact with the pericardium. Locomotion was achieved across several surfaces including the anterior wall of the beating right ventricle, the anterolateral wall of the beating left ventricle, and the anterior wall of the left atrial appendage. The trials were recorded through the translucent pericardium using an external handheld video camera (Fig. 2).

Myocardial injections of tissue-marking dye $(0.5 \mathrm{cc})$ were performed at two locations: the bifurcation of the left anterior descending coronary artery and the takeoff of the diagonal branch, and the diagonal coronary artery. In each case, HeartLander walked to the planned site and locked down with suction on both pads, then the surgeon advanced the needle into the myocardium and performed the injection. The targets were visual located and recorded using the SVP, due to malfunctioning of the HeartLander visual system (Fig. 3A). No

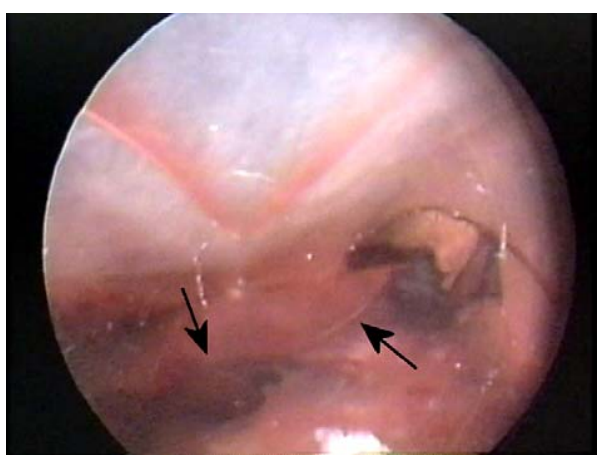

A

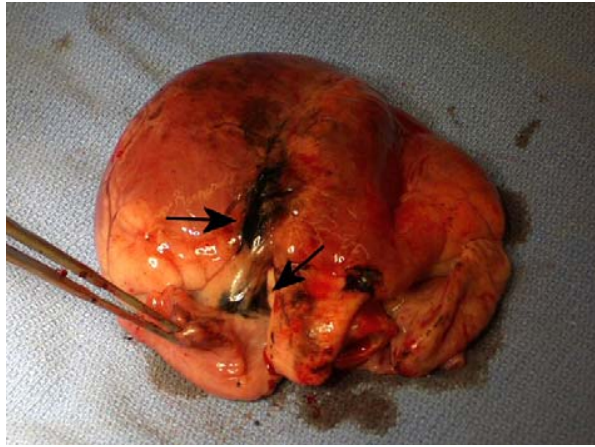

B

Fig. 3. (A) HeartLander performing a myocardial injection of tissue marking dye, as viewed beneath the pericardium by the SVP device. The right arrow highlights the needle, while the left arrow highlights the dyed myocardial tissue. (B) The excised porcine heart, with arrows highlighting the two injection sites. 
bleeding was observed following needle withdrawal. Confirmation of successful injection was made during postoperative examination of the excised porcine hearts (Fig. 3B). Following all procedures, the surgeon confirmed that no damage was done to the epicardium or pericardium.

\section{Discussion}

The results presented herein demonstrate the feasibility of using a mobile robot to provide access and a stable platform for myocardial injections on an exposed beating heart with the pericardium intact. Future research will focus on improvements in miniaturization and flexibility in order to allow the device to travel effectively to any location on the heart during closed-chest testing. In order to eliminate the rigidity of the fiberscope, an alternative video system will soon be implemented using an onboard camera. New prototypes will be developed to allow porcine tests to proceed from open-heart testing to closed-chest testing using a subxiphoid approach. As clinical testing of cardiomyoplasty increases in the near future, techniques for precisely and effectively delivering intramyocardial injections of stem cells in a minimally invasive manner will be crucial. The HeartLander research and development presents a viable method to achieve this goal.

\section{References}

[1] M.S. Lee, M. Lill, R.R. Makkar, Stem cell transplantation in myocardial infarction, Rev. Cardiovasc. Med. 5 (2004) $82-98$.

[2] M. Grogan, et al., Long-term outcome of patients with biopsy proved myocarditis: comparison with idiopathic dilated cardiomyopathy, J. Am. Coll. Cardiol. 26 (1995) 80-84.

[3] K.C. Wollert, et al., Bone marrow transfer to enhance ST-elevation infarct regeneration (BOOST) trial, Late Breaking Clinical Trials, American Heart Association, 2003.

[4] M. Brehm, et al., Angiogenesis and myogenesis after intracoronary transplantation of autologous bone marrow cells in patients with acute myocardial infarction (abstract), Circulation 108 (2003) 1929.

[5] A.N. Patel, et al. First heart failure patients ever to receive stem cells in minimally invasive procedure have improved heart function. The 41st Annual Meeting of the Society for Thoracic Surgery, in press.

[6] N.A. Patronik, M.A. Zenati, C.N. Riviere, Preliminary evaluation of a tethered robotic device for navigation on the beating heart. Comput. Aided Surg. (in press), Invited Paper.

[7] M. Zenati, A. Chin, D. Schwartzman, Subxiphoid videopericardioscopy: a new minimally invasive approach to the pericardial cavity for epicardial therapies, Proceedings of ISMICS 7th Annual Scientific Meeting, Heart Surg Forum, vol. 7, 2004, pp. 639-643.

[8] G. Knight, W.D. Fox, D.R. Schulze, Cardiac stabilizer device having multiplexed vacuum ports and method of stabilizing a beating heart, U.S. Patent 6,589,166 (2002). 